\title{
Polycyclic Aromatic Hydrocarbon Risk Assessment and Analytical Methods Using QuEchERS Pretreatment for the Evaluation of Herbal Medicine Ingredients in Korea
}

\author{
Hee-Jeong Hwang ${ }^{1}$, Sae-Ha Lee ${ }^{2}$, Yong-Yeon Kim ${ }^{2} \mathbb{D}$ and Han-Seung Shin ${ }^{2, *}$ \\ 1 Research Institute of Biotechnology and Medical Converged Science, Dongguk University-Seoul, 32, \\ Dongguk-ro, Ilsandong-gu, Goyang-si 10326, Gyeonggi-do, Korea; piatop@hanmail.net \\ 2 Department of Food Science and Biotechnology, Dongguk University-Seoul, 32, Dongguk-ro, Ilsandong-gu, \\ Goyang-si 10326, Gyeonggi-do, Korea; yshwl11@naver.com (S.-H.L.); kimyy613@naver.com (Y.-Y.K.) \\ * Correspondence: spartan@dongguk.edu; Tel.: +82-31-961-5184
}

\section{check for} updates

Citation: Hwang, H.-J.; Lee, S.-H.; Kim, Y.-Y.; Shin, H.-S. Polycyclic Aromatic Hydrocarbon Risk Assessment and Analytical Methods Using QuEchERS Pretreatment for the Evaluation of Herbal Medicine Ingredients in Korea. Foods 2021, 10, 2200. https://doi.org/10.3390/foods 10092200

Academic Editors: Federico Marini and Alessandra Biancolillo

Received: 18 August 2021

Accepted: 13 September 2021

Published: 16 September 2021

Publisher's Note: MDPI stays neutral with regard to jurisdictional claims in published maps and institutional affiliations.

Copyright: (c) 2021 by the authors. Licensee MDPI, Basel, Switzerland. This article is an open access article distributed under the terms and conditions of the Creative Commons Attribution (CC BY) license (https:/ / creativecommons.org/licenses/by/ $4.0 /)$.

\begin{abstract}
Polycyclic aromatic hydrocarbons (PAHs) are carcinogenic and mutagenic compounds that are often formed during the thermal processing of herbal medicine ingredients. In this study, the concentrations of four PAHs (PAH4) in various herbal medicine ingredients were monitored. Further, the QuEChERS method was used to replace conventional pretreatment, a more complex and cumbersome approach. The recovery range of the QuEChERS method ranged between 89.65-118.59\%, and the average detection levels of benzo[a]anthracene (BaA), chrysene (CHR), benzo[b]fluoranthene $(\mathrm{BbF})$, and Benzo[a]pyrene $(\mathrm{BaP})$ in 50 herbal medicine ingredients were $0.18,0.27,1.13$, and $0.17 \mu \mathrm{g} / \mathrm{kg}$, respectively. The $\mathrm{BaP}$ and PAH4 levels in all tested samples were deemed safe according to risk characterization analyses based on European Union and Korean guidelines. Therefore, our findings indicated that the QuEChERS method could be used as an effective alternative to conventional sample pretreatment for the analysis of herbal medicine ingredients.
\end{abstract}

Keywords: polycyclic aromatic hydrocarbons; herbal medicine; QuEChERS; exposure assessment; risk characterization

\section{Introduction}

Although synthetic drugs were proven to be effective treatments for various diseases, interest in natural therapeutic products steadily increased throughout recent years. Particularly, there is a growing interest in herbal medicines that were used for approximately 5000 years as traditional remedies. Nevertheless, there are also growing concerns regarding the carcinogenic compounds that are unintentionally generated during the heat treatment process of herbal medicine ingredients, particularly polycyclic aromatic hydrocarbons (PAHs). PAHs can be generated from the incomplete combustion of organic matter or can be produced when organic sediments are chemically transformed into fossil fuels such as oil and coal [1]. PAHs are formed in two steps: pyrolysis and pyrosynthesis. Organic materials become partially decomposed and divided into unstable fractions when they reach a high temperature (pyrolysis). These fractions are then recombined to become extremely reactive radicals, which are stable PAHs (pyrosynthesis) [2]. PAHs are formed during the combustion process of carbonaceous materials at high temperatures [3]. Applying high temperatures to herbal medicine ingredients during the manufacturing process can thus lead to PAH generation. Also, relocation of PAHs to the raw material in environments such as soil, air, and so on can be involved in the detection of PAHs.

PAHs pose a severe risk to human and environmental health. Furthermore, these compounds are highly pervasive and persistent, and thus, remain in the environment for long periods by interacting with particles in the soil, sediment, and air, resulting in severe pollution. PAHs with two or more aromatic rings in a linear, cluster, or angular arrangement were linked to tumorigenesis in humans [4]. Therefore, the International 
Agency for Research on Cancer (IARC) classified PAHs as a working group to evaluate their carcinogenicity to humans [5]. Benzo[a]pyrene (BaP) is considered a Group1 compound (carcinogenic to humans), whereas benzo[a]anthracene (BaA), chrysene (CHR), and benzo[b]fluoranthene $(\mathrm{BbF})$ are classified as Group2B (possibly carcinogenic to humans). In December 2009, the Korea Food \& Drug Administration (KFDA) established the benzopyrene standard for all herbal medicines except for mineral herbal medicines to be less than $5 \mu \mathrm{g} / \mathrm{kg}$, and they also announced a test method [6].

PAH analyses are conducted in many areas, particularly the food industry; however, sample pretreatment is time-consuming and highly inconvenient. Determination of $\mathrm{PAH}$ in herbs, tea, and edible oils are often carried out using gas chromatography (GC) and high-performance liquid chromatography (HPLC) [7-9]. To address these methodological challenges, the quick, easy, cheap, effective, rugged, and safe (QuEChERS) method was developed [10]. This approach has become one of the most commonly used methods for the analysis of multiclass pesticide residues in vegetables and fruits, and more recently was used to detect traces of contaminants such as PAHs [8,11,12]. This study sought to monitor four PAHs (PAH4) contents in herbal medicine ingredients using the QuEChERS method coupled with HPLC-FLD and evaluate the efficiency of this approach by comparing it with that of the conventional pretreatment method coupled with GC/MS. Further, risk assessment analyses were conducted by calculating toxicity equivalency (TEQ), the daily average intake (DAI), and margin of exposure (MOE) to evaluate cancer risks in the Korean population.

\section{Materials and Methods}

\subsection{Chemicals and Materials}

The PAH4 standards benzo[a]pyrene (BaP, CAS No. 50-32-8), benzo[b]fluoranthene (BbF, CAS No. 205-99-2), chrysene (CHR, CAS No. 218-01-9), and benzo[a]anthracene (BaA, CAS No. 56-55-3) and internal standards (IS) 3-methylcholanthrene (CAS No. 56-49-5), chrysene-d12 (CAS No. 1719-03-5), and benzo[a]pyrene-d12 (CAS No. 63466-71-7) were obtained from Supelco, Inc. (Bellefonte, PA, USA). All materials and chemicals were of GC and HPLC analytical grade. Acetonitrile (ACN) (CAS No. 75-05-8), dichloromethane (DCM) (CAS No. 75-09-2), methanol (CAS No. 67-56-1), and hexane (CAS No. 110-54-3) were obtained from Burdick \& Jackson (Muskegon, MI, USA). Sodium sulfate anhydrous $\left(\mathrm{Na}_{2} \mathrm{SO}_{4}\right.$ ) (CAS No. 7757-82-6) 99\% w/w purity; (Samchun Co., Ltd., Seoul, Korea) was used for dehydration. Distilled water (CAS No. 7732-18-5) and N,N-dimethylformamide (CAS No. 68-12-2) were prepared using a Milli-Q water purification system (Millipore, Billerica, MA, USA). Kovax syringes ( $3 \mathrm{~mL}$ ) were obtained from Korea Vaccine Co., Ltd. (Gyeonggi-do, Korea). Polytetrafluoroethylene (PTFE) membrane filters $(0.45 \mathrm{~mm})$ were purchased from Advantec Co., Ltd. (Chiyoda City, Japan). Sep-Pak Florisil cartridges (Waters Corp., Milford, MA, USA) were utilized for solid-phase extraction (SPE).

\subsection{Sample Preparation for PAH4 Evaluation}

From January to September 2019, 50 types of herbal medicine products were provided by the Ministry of Food and Drug Safety (MFDS) in the Republic of Korea to evaluate PAH4 contents. The samples included milk-thistle fruit, fresh bilberry fruit, ginkgo leaf, angelica gigas root, grape seed, chaenomelis fructus, saposhnikovia root, dipsaci radix, acanthopanax root bark, archyranthes root, clematis radix, cinnamon bark, gentianae macrophyllae radix, cnidium rhizome, gastrodia rhizome, safflower, Vitis vinifera seed, and ivy leaf, among others. The samples were chopped into small-sized pieces and stored at $-18^{\circ} \mathrm{C}$ prior to the analysis.

\subsection{Extraction and Clean-Up}

The homogenized herbal medicinal samples were weighted $(5 \mathrm{~g})$ in round bottom flasks containing $100 \mathrm{~mL}$ of water for ultrasonic extraction $(60 \mathrm{~min})$. After this water ultrasonic extraction step, hexane $(100 \mathrm{~mL})$ was added for an additional ultrasonic ex- 
traction (60 min) and spiked with $1.0 \mathrm{~mL}$ of $50 \mu \mathrm{g} / \mathrm{kg}$ IS (3-methylcholanthrene, BaP-d12, and CHR-d12). For liquid-liquid extraction, the mixtures were centrifuged at $3200 \mathrm{~g}$ for $10 \mathrm{~min}$, and the upper hexane layer was transferred to a separatory funnel. After liquidliquid extraction, $50 \mathrm{~mL}$ of $\mathrm{N}, \mathrm{N}$-dimethylformamide/water $(9: 1 ; v / v)$ was added to the hexane layer of the separatory funnel, after which the $\mathrm{N}, \mathrm{N}$-dimethylformamide/water $(9: 1 ; v / v)$ layer was transferred to another separatory funnel after shaking; this extraction procedure was repeated three times. Next, $100 \mathrm{~mL}$ of $1 \%$ sodium sulfate solution and $50 \mathrm{~mL}$ of hexane were added to the separatory funnel, after which the separated hexane layer was transferred to another separatory funnel; thirty-five $\mathrm{mL}$ of hexane was added to the separatory funnel to extract with shaking. This hexane layer was recovered, and the extraction procedure was repeated twice. The hexane layer was then washed with $50 \mathrm{~mL}$ of water and conjugated via dehydrating filtration in $30 \mathrm{~g} \mathrm{of} \mathrm{Na}_{2} \mathrm{SO}_{4}$ in a round-bottomed flask. The extract was concentrated to $2 \mathrm{~mL}$ in a water bath at $45^{\circ} \mathrm{C}$ under reduced pressure $(70 \mathrm{kPa})$ in a vacuum rotary evaporator.

Purification was carried out after the extraction process. Firstly, the concentrated extract was separated from the impurities via solid-phase extraction using Sep-Pak Florisil cartridges (Waters Corp., Milford, MA, USA) preconditioned with $10 \mathrm{~mL}$ of DCM and $20 \mathrm{~mL}$ of hexane eluted at a rate of 2 drops per second. Next, the extract was eluted from the SPE cartridge with $15 \mathrm{~mL}$ of DCM and $5 \mathrm{~mL}$ of hexane:DCM $(3: 1 ; v / v)$. This eluted solution was dried in a heating block at $35^{\circ} \mathrm{C}$ under a constant nitrogen gas flow. The residues were dissolved with $1 \mathrm{~mL}$ of $\mathrm{ACN}$ by vortexing. The testing solution was filtered with a $0.45-\mu \mathrm{m}$ PTFE membrane syringe filter and transferred to amber vials with a screw cap for HPLC-FLD and GC-MS analyses.

\subsection{Preprocessing of Samples Using QuEChERS}

The QuEChERS method entails an extraction step and a clean-up step. For the extraction step, we employed the QuEChERS Performance Standards Kit (Restek, GmbH Schaberweg 23, Bad Homburg, Germany; Cat. No. \#25847) containing 4 g $\mathrm{MgSO}_{4}$ for dehydration and $1 \mathrm{~g} \mathrm{NaCl}$ to maintain the $\mathrm{pH}$. For preprocessing, the samples were homogenized to increase their surface area. The homogenized $1 \mathrm{~g}$ of samples and $10 \mathrm{~mL}$ of distilled water were transferred to a $50 \mathrm{~mL}$ conical tube (Supermax Corporation Berhad, Kuala Lumpur, Malaysia; Cat. No. \#S20050). After $1 \mathrm{~h}, 10 \mathrm{~mL}$ of hexane:acetone $(1: 1 ; v / v)$ was added and the mixture was vortexed for $10 \mathrm{~min}$. After adding the QuEChERS salts, the samples were centrifuged at $3000 \mathrm{~g}$ for $5 \mathrm{~min}$. For the clean-up step, Resprep silica SPE cartridges (Restek, GmbH Schaberweg 23, Bad Homburg, Germany; Cat. No. \#24036) were used to extract hydrophilic analytes from nonpolar matrices. The silica cartridge was rinsed with $3 \mathrm{~mL}$ of methanol and $3 \mathrm{~mL}$ of acetone under high vacuum conditions with 3 $\mathrm{mL}$ of hexane:methylene chloride $(1: 1 ; v / v)$ and $6 \mathrm{~mL}$ of hexane at a 1 drop per second rate. The supernatant in the conical tube was then transferred to a conditioned silica cartridge, followed by $5 \mathrm{~mL}$ of hexane:methylene chloride $(85: 15 ; v / v)$ solution. This extract was evaporated using a gentle stream of nitrogen gas in a heating block at $50^{\circ} \mathrm{C}$. Finally, $1 \mathrm{~mL}$ of acetonitrile was used to dissolve the extract for HPLC-FLD analyses.

\subsection{GC-MS Analysis of PAH4}

PAH4 in the samples processed via the conventional pretreatment method was analyzed using a GC-MS system (Agilent Technologies 7820A/5975 MSD GC-MS system, Santa Clara, CA, USA) equipped with an HP-5MS UI capillary column $(0.25 \mathrm{~mm} \times 30 \mathrm{~m}$ i.d., $0.25 \mu \mathrm{m}$ particle size). Helium gas $(99.99 \%)$ was used as a carrier at a constant flow rate of $1.0 \mathrm{~mL} / \mathrm{min}$. The oven temperature was set to $80^{\circ} \mathrm{C}$, held for $1 \mathrm{~min}$, increased to $245^{\circ} \mathrm{C}$ at a $10{ }^{\circ} \mathrm{C} / \mathrm{min}$ rate, then to $290^{\circ} \mathrm{C}$ at a $10{ }^{\circ} \mathrm{C} / \mathrm{min}$ rate, and finally held postrun at $310^{\circ} \mathrm{C}$ for $5 \mathrm{~min}$. The sample solution was injected in splitless mode at $310^{\circ} \mathrm{C}$, and the injection volume was $1.0 \mathrm{~mL}$. The quadrupole temperature was $150{ }^{\circ} \mathrm{C}$ and the MS source temperature was $250^{\circ} \mathrm{C}$. Each of the IS and the PAH4 had two qualifier ions and one target ion (underlined). The selected ions were 228, 226, and 229 for CHR and BaA; 
252, 250, and 253 for BaP and BbF; 240, 236, and 241 for CHR-d12; and 264, 263, and 265 for $\mathrm{BaP}-\mathrm{d} 12$. PAHs were identified via extracted ion chromatogram, and the molecular mass was determined using the GC-MS program.

\subsection{HPLC-FLD Analysis of PAH4}

PAH4 in the samples treated via the QuEChERS pretreatment method were analyzed using an HPLC-FLD analysis instrument (Dionex U3000 HPLC coupled with a fluorescence detector, Thermoherbal Medicineer, Sunnyvale, CA, USA) equipped with a ZORBAX eclipse C18 plus column (4.6 mm id $\times 250 \mathrm{~mm} \times 5 \mu \mathrm{m}$, Agilent, Santa Clara, CA, USA). The injection volume was $10 \mu \mathrm{m}$ at a $1.0 \mathrm{~mL} / \mathrm{min}$ flow rate. Acetonitrile and water were used as the mobile phase at a 65:35 (acetonitrile:water; \%) ratio from 0 to $20 \mathrm{~min}$ and 70:30 (\%) from 20 to $60 \mathrm{~min}$. The excitation and emission wavelengths were $245 / 390 \mathrm{~nm}$ from 0 to $30 \mathrm{~min}$ and $294 / 404 \mathrm{~nm}$ from 30 to $60 \mathrm{~min}$. Separation was conducted with the following gradient program: 35\% B for $20 \mathrm{~min}$ and $30 \% \mathrm{~B}$ for the last $30 \mathrm{~min}$.

\subsection{Identification and Quantification of PAH4}

PAH4 were identified by comparing their retention times and those of standards. For validation, 5 concentrations of the PAH4 solutions $(3,5,10,20$, and $40 \mu \mathrm{g} / \mathrm{kg})$ containing $50 \mu \mathrm{g} / \mathrm{kg}$ of the IS mixture were evaluated.

\subsection{Method Validation for Analytical Quality Assurance}

All analytical methods were validated for limit of detection (LOD), limit of quantification (LOQ), linearity, precision (\%), and recovery (\%) on herbal medicine samples. Calibration curves were created from $50 \mu \mathrm{g} / \mathrm{kg}$ of IS mixture (3-methylcholanthrene; CHR$\mathrm{d} 12$ and BaP-d12) and PAH4 standard mixtures at 3, 5, 10, 20, and $40 \mu \mathrm{g} / \mathrm{kg}$.

\subsection{Application of TEQ Concentration}

$\mathrm{BaP}$ is the most widely recognized PAH and Class1 carcinogenic compound. Human exposure was evaluated by analyzing herbal medicine products spiked with 3-methylcholanthrene, CHR-d12, and BaP-d12. This study was conducted to estimate the PAH exposure levels of the entire Korean population.

The relative toxicity coefficient of congener $i$ based on BaP cancer potency (Toxic equivalency factor, TEFs) was estimated as BaP equivalents, considering that each PAH has a different toxicity level. BaP concentration conversion was conducted using TEQ by multiplying each PAH by their respective TEF:

$$
\mathrm{TEQ}=\sum_{i=1}^{n}[C i] \times \mathrm{TEF} i
$$

where $\mathrm{C} i$ is the concentration of each PAH congener in herbal medicine products, and TEF $i$ is the relative toxicity coefficient of congener $i$ based on $\mathrm{BaP}$ cancer potency corresponding to $\mathrm{BaP}$.

TEQ data were acquired from the $\mathrm{C} i$ of PAHs among many herbal medicine products based on analytical methods and the TEFi proposed by [13] (i.e., the latest study on herbal medicine products). Given the differences in sample numbers for each herbal medicine product, the average concentrations of PAH4 in herbal medicine products were not determined.

\subsection{Exposure Assessment}

Dietary intake is the main route of PAH exposure in humans. Therefore, PAH exposure in adults and children was estimated based on oral consumption of leading herbal medicine products. 
Daily exposure to PAH4 was calculated based on total lifetime intake. By applying the $\mathrm{BaP}$ equivalent concentrations, $\mathrm{PAH} 4$ concentrations, and the daily consumption of herbal medicine products, the DAI was calculated as follows:

$$
\operatorname{DAI}(\mu \mathrm{g} / \mathrm{kg} / \text { day })=\sum_{i=1}^{n} \frac{C i \times I R i \times \mathrm{ED}}{\mathrm{BW} \times \mathrm{AT}},
$$

where $C i$ is the respective TEQ of the PAH4 in herbal medicine $i(\mu \mathrm{g} / \mathrm{kg}) ; I R i$ is the average daily intake of herbal medicine $i$ according to the National Health and Nutrition Survey (ingestion rate, $0.0061 \mathrm{~g} /$ day); AT is the average life expectancy (80.4 years); BW is the body weight by age group ( $64 \mathrm{~kg}$ ); ED is the exposure period (45 years). The AT and BW values were collected from the 2018 statistics data of Korea [13] and the 2018 National Health Screening Statistical Yearbook of Korea.

\subsection{Exposure Assessment}

To assess risk, the MOE was calculated based on the benchmark dose lower confidence limit (BMDL) $(\mathrm{mg} / \mathrm{kg} \cdot \mathrm{BW} /$ day) and the dietary exposure $(\mathrm{mg} / \mathrm{kg} \cdot \mathrm{BW} /$ day). $\mathrm{CB} i$ is the concentration of $\mathrm{BaP}(\mathrm{mg} / \mathrm{kg})$.

$$
\begin{gathered}
\text { Dietary exposure }=\sum_{i=1}^{n} \frac{C B i \times I R i}{\mathrm{BW}}, \\
\mathrm{MOE}=\frac{\mathrm{BMDL}}{\text { Dietary exposure }},
\end{gathered}
$$

The Committee on Carcinogenicity of Chemicals in herbal medicine, Consumer Products, and the Environment classified the risk of MOE values $<10,000$ as "possible concern," $10,000-1,000,000$ as "low concern," $>100,000$ as "negligible concern with action minimizing future exposure," and $>1,000,000$ as "negligible concern" [14]. In other words, the level of concern decreases as the MOE value increases.

Excessive cancer risk was estimated based on the cancer risk of BaP and the DAI values as follows:

$$
\text { Excessive cancer risk }=\text { cancer risk of } \mathrm{BaP} \times \mathrm{DAI} \text {, }
$$

where BaP's cancer potency is $7.3(\mathrm{mg} / \mathrm{kg} / \text { day })^{-1}$ based on the U.S. Environmental Protection Agency's integrated risk information system. Excessive cancer risk values $>10^{-4}$ are considered a "serious risk," those between $10^{-6}-10^{-4}$ are considered a "potential risk," and those $<10^{-6}$ are considered "safe and acceptable."

\subsection{Statistical Analyses}

All analyses were conducted in triplicate and the data were expressed as mean \pm standard deviation (SD) using Microsoft Excel (version 2016, Microsoft, Redmond, WA, USA).

\section{Results}

\subsection{Method Validation and Confirmation of PAH4 in Herbal Medicine}

The GC-MS and HPLC chromatograms of PAH4 standards (A), PAH4 with spiked sample (B), two internal standards with a blank sample (C), and chromatograms of PAHs for each sample (D) are presented in Figures S1 and S2. A calibration curve was generated based on five different standard mixture concentrations $(3,5,10,20,40 \mu \mathrm{g} / \mathrm{kg})$ for the validation of the conventional sample pretreatment method via GC-MS. The linearity, LOD, and LOQ of the PAH4 are summarized in Table 1 . All correlation coefficients $\left(R^{2}\right)$ values for PAH4 exceeded 0.99 , and the LOD and LOQ values were $0.08-0.15 \mu \mathrm{g} / \mathrm{kg}$ and 0.24- $0.45 \mu \mathrm{g} / \mathrm{kg}$, respectively. Likewise, regarding the validation of the QuEChERS pretreatment method coupled with HPLC-FLD, the correlation coefficient $\left(R^{2}\right)$ exceeded 0.99 
for PAH4 at all concentrations. The LOD and LOQ values ranged from $0.08-0.17 \mu \mathrm{g} / \mathrm{kg}$ and from $0.25-0.51 \mu \mathrm{g} / \mathrm{kg}$, respectively.

Table 1. Comparison of typical sample treatment and QuEChERS in regard with linearity with equation of calibration, limit of detection (LOD), and limit of quantification (LOQ) of PAH4 in herbal medicine.

\begin{tabular}{|c|c|c|c|c|c|}
\hline PAHs & Tretment & Linearity ${ }^{(1)}$ & $\mathbf{R}^{2}$ & $\operatorname{LOD}_{(2)}^{(\mu \mathrm{g} / \mathrm{kg})}$ & LOQ $\underset{(3)}{(\mu \mathrm{g} / \mathrm{kg})}$ \\
\hline \multirow{2}{*}{$\mathrm{BaA}$} & Typical & $y=0.0245 x-0.0054$ & 0.999 & 0.14 & 0.43 \\
\hline & QuEChERS & $y=0.0115 x+0.0075$ & 0.999 & 0.12 & 0.37 \\
\hline \multirow[b]{2}{*}{ CHR } & Typical & $y=0.018 x+0.0026$ & 0.999 & 0.14 & 0.42 \\
\hline & QuEChERS & $y=0.0104 x+0.0009$ & 0.999 & 0.17 & 0.51 \\
\hline \multirow{2}{*}{$\mathrm{BbF}$} & Typical & $y=0.00379 x+0.0088$ & 0.998 & 0.15 & 0.45 \\
\hline & QuEChERS & $\mathrm{y}=0.0027 \mathrm{x}+0.0002$ & 0.999 & 0.14 & 0.41 \\
\hline \multirow{2}{*}{$\mathrm{BaP}$} & Typical & $y=0.0273 x+0.0075$ & 0.999 & 0.08 & 0.24 \\
\hline & QuEChERS & $y=0.0183 x-0.0003$ & 0.999 & 0.08 & 0.25 \\
\hline
\end{tabular}

(1) Numbers express the mean values $(n=3) .{ }^{(2)}$ Set up in a signal-to-noise ratio $(S / N)=3.3 .{ }^{(3)}$ Set up in a signal-to-noise ratio $(\mathrm{S} / \mathrm{N})=10$.

Further, as shown in Table 2, recovery and precision were evaluated by repeating all five concentrations three times within a day (intraday) and once again for two days (interday). When using the conventional sample treatment method, the recovery and precision values for the intraday experiment were $93.19-117.26 \%$ and $0.04-7.84 \%$, respectively. For the interday experiment, the recovery values varied from $91.51-119.51 \%$, whereas precision varied from $0.11-5.71 \%$. The intraday recovery and precision values for the QuEChERS method were $89.65-118.59 \%$ and $0.17-2.15 \%$, respectively. Interday recovery and precision varied from $95.31-117.87 \%$ and from $0.09-9.38 \%$, respectively.

Table 2. Recovery and precision comparison of typical sample treatment and QuEChERS for PAH4 in herbal medicine ingredients.

\begin{tabular}{|c|c|c|c|c|c|c|c|c|c|}
\hline \multirow{3}{*}{ PAHs } & \multirow{3}{*}{$\begin{array}{l}\text { Concentration } \\
(\mu \mathrm{g} / \mathrm{kg})\end{array}$} & \multicolumn{4}{|c|}{ Intraday $(n=3)$} & \multicolumn{4}{|c|}{ Interday $(n=3)$} \\
\hline & & \multicolumn{2}{|c|}{$\begin{array}{c}\text { Recovery } \\
(\%)\end{array}$} & \multicolumn{2}{|c|}{$\begin{array}{l}\text { Precision } \\
(\%)\end{array}$} & \multicolumn{2}{|c|}{$\begin{array}{l}\text { Recovery } \\
(\%)\end{array}$} & \multicolumn{2}{|c|}{$\begin{array}{l}\text { Precision } \\
(\%)\end{array}$} \\
\hline & & Typ & QuE & Typ & QuE & Typ & QuE & Typ & QuE \\
\hline \multirow{5}{*}{$\mathrm{BaA}$} & 3 & 110.93 & 89.65 & 0.61 & 0.45 & 101.99 & 104.67 & 1.34 & 9.38 \\
\hline & 5 & 113.38 & 111.22 & 0.25 & 0.82 & 119.24 & 117.81 & 2.83 & 0.69 \\
\hline & 10 & 116.83 & 111.17 & 0.4 & 0.85 & 119.51 & 111.74 & 0.87 & 0.65 \\
\hline & 20 & 110.69 & 102.09 & 0.46 & 0.44 & 116.72 & 108.74 & 0.11 & 0.5 \\
\hline & 40 & 112.68 & 109.68 & 0.37 & 0.28 & 113.1 & 110.08 & 1.05 & 0.59 \\
\hline \multirow{5}{*}{ CHR } & 3 & 109.79 & 102.7 & 1.11 & 2.15 & 109.88 & 98.34 & 1.23 & 3.63 \\
\hline & 5 & 104.78 & 118.59 & 1.09 & 0.46 & 106.15 & 117.87 & 0.84 & 0.09 \\
\hline & 10 & 105.98 & 109.8 & 0.04 & 0.91 & 106.35 & 110.96 & 0.33 & 0.51 \\
\hline & 20 & 104.65 & 103.57 & 0.1 & 0.69 & 105.22 & 107.58 & 0.66 & 1.38 \\
\hline & 40 & 101.73 & 109.47 & 0.47 & 0.46 & 102.21 & 108.91 & 1.08 & 0.74 \\
\hline \multirow{5}{*}{$\mathrm{BbF}$} & 3 & 101.2 & 99.69 & 7.84 & 0.19 & 103.73 & 103.63 & 5.71 & 6.9 \\
\hline & 5 & 117.26 & 112.57 & 1.47 & 1.41 & 114.16 & 114.54 & 3.00 & 4.05 \\
\hline & 10 & 107.69 & 103.5 & 0.64 & 0.84 & 113.6 & 103.77 & 5.06 & 3.48 \\
\hline & 20 & 106.83 & 105.45 & 0.38 & 0.32 & 108.42 & 105.4 & 3.32 & 0.87 \\
\hline & 40 & 95.42 & 104.12 & 0.69 & 0.58 & 93.75 & 104.83 & 2.97 & 0.65 \\
\hline \multirow{5}{*}{$\mathrm{BaP}$} & 3 & 111.33 & 99.44 & 6.67 & 1.01 & 109.58 & 95.31 & 1.85 & 1.00 \\
\hline & 5 & 112.57 & 100.21 & 1.31 & 0.27 & 109.20 & 101.06 & 5.52 & 1.58 \\
\hline & 10 & 98.9 & 103.79 & 1.5 & 0.51 & 100.14 & 106.45 & 0.54 & 2.42 \\
\hline & 20 & 98.63 & 100.22 & 0.2 & 0.68 & 100.37 & 99.69 & 1.71 & 0.35 \\
\hline & 40 & 93.19 & 103.16 & 0.55 & 0.17 & 91.51 & 103.68 & 2.5 & 0.64 \\
\hline
\end{tabular}




\subsection{Comparison of the Two Different Sample Pretreatment Methods for the Analysis of Herbal Medicine Ingredients}

PAH4 in herbal medicine ingredients was monitored with both the conventional and QuEChERS methods after each of the parameters for method validation was evaluated. An established validation method was employed for the simultaneous determination of PAH4 in 50 herbal medicine ingredients. Tables 3 and 4 summarize the PAH4 concentrations in samples obtained using two different sample pretreatment methods: (1) conventional sample pretreatment method using GC/MS, and (2) QuEChERS coupled with HPLC-FLD. When using the conventional pretreatment method, the $\mathrm{BaA}, \mathrm{CHR}, \mathrm{BbF}$, and $\mathrm{BaP}$ detection ranges were 0-0.40 $\mu \mathrm{g} / \mathrm{kg}, 0-0.46 \mu \mathrm{g} / \mathrm{kg}, 0-1.84 \mu \mathrm{g} / \mathrm{kg}$, and $0-0.27 \mu \mathrm{g} / \mathrm{kg}$, respectively. The QuEChERS method rendered $\mathrm{BaA}, \mathrm{CHR}, \mathrm{BbF}$, and $\mathrm{BaP}$ detection ranges of $0-0.63 \mu \mathrm{g} / \mathrm{kg}, 0-0.82 \mu \mathrm{g} / \mathrm{kg}, 0.26-3.23 \mu \mathrm{g} / \mathrm{kg}$, and 0-0.48 $\mu \mathrm{g} / \mathrm{kg}$, respectively. When using the conventional pretreatment method, $\mathrm{BaA}, \mathrm{CHR}, \mathrm{BbF}$, and $\mathrm{BaP}$ were detected in 41,41 , 30 , and 21 samples out of 50, whereas this compound was detected in 21, 41, 49, and 41 samples out of 50 when the QuEChERS method was used. Regardless of the type of pretreatment method, sample 32 showed the highest concentration of $\mathrm{BaA}, \mathrm{CHR}, \mathrm{BbF}$, and $\mathrm{BaP}$. Further, the PAH4 value obtained by combining the concentrations of the four substances was $2.97 \mu \mathrm{g} / \mathrm{kg}$ when using the conventional pretreatment method and $5.16 \mu \mathrm{g} / \mathrm{kg}$ when using the QuEChERS method.

Table 3. PAH4 concentration in herbal medicines analyzed using typical pretreatment method.

\begin{tabular}{|c|c|c|c|c|c|}
\hline \multirow{2}{*}{ Sample } & \multicolumn{5}{|c|}{ Concentration $(\mu \mathrm{g} / \mathrm{kg})^{(1)}$} \\
\hline & BaA & CHR & $\mathrm{BbF}$ & BaP & PAH4 \\
\hline Sample 1 & N.D. ${ }^{(2)}$ & N.D. & N.D. & N.D. & N.D. \\
\hline Sample 2 & $0.07 \pm 0.00$ & $0.01 \pm 0.00$ & N.D. & N.D. & 0.08 \\
\hline Sample 3 & $0.07 \pm 0.00$ & $0.01 \pm 0.00$ & N.D. & N.D. & 0.09 \\
\hline Sample 4 & $0.32 \pm 0.01$ & $0.35 \pm 0.01$ & $1.34 \pm 0.04$ & $0.2 \pm 0.01$ & 2.22 \\
\hline Sample 5 & $0.07 \pm 0.00$ & $0.01 \pm 0.00$ & N.D. & N.D. & 0.08 \\
\hline Sample 6 & $0.39 \pm 0.04$ & $0.44 \pm 0.06$ & $1.78 \pm 0.28$ & $0.26 \pm 0.04$ & 2.87 \\
\hline Sample 7 & $0.12 \pm 0.00$ & $0.07 \pm 0.01$ & N.D. & $0.01 \pm 0.00$ & 0.19 \\
\hline Sample 8 & $0.19 \pm 0.00$ & $0.17 \pm 0.01$ & $0.46 \pm 0.02$ & $0.07 \pm 0.00$ & 0.88 \\
\hline Sample 9 & $0.10 \pm 0.00$ & $0.04 \pm 0.00$ & N.D. & N.D. & 0.14 \\
\hline Sample 10 & $0.30 \pm 0.01$ & $0.32 \pm 0.01$ & $1.21 \pm 0.06$ & $0.18 \pm 0.01$ & 2.01 \\
\hline Sample 11 & $0.28 \pm 0.02$ & $0.30 \pm 0.02$ & $1.08 \pm 0.11$ & $0.16 \pm 0.02$ & 1.82 \\
\hline Sample 12 & $0.08 \pm 0.00$ & $0.01 \pm 0.00$ & N.D. & N.D. & 0.09 \\
\hline Sample 13 & $0.07 \pm 0.00$ & $0.01 \pm 0.00$ & N.D. & N.D. & 0.08 \\
\hline Sample 14 & $0.07 \pm 0.00$ & $0.01 \pm 0.00$ & N.D. & N.D. & 0.08 \\
\hline Sample 15 & $0.29 \pm 0.01$ & $0.30 \pm 0.02$ & $1.09 \pm 0.09$ & $0.16 \pm 0.01$ & 1.84 \\
\hline Sample 16 & $0.07 \pm 0.00$ & $0.01 \pm 0.00$ & N.D. & N.D. & 0.08 \\
\hline Sample 17 & $0.31 \pm 0.01$ & $0.33 \pm 0.01$ & $1.23 \pm 0.04$ & $0.18 \pm 0.01$ & 2.04 \\
\hline Sample 18 & $0.30 \pm 0.01$ & $0.32 \pm 0.01$ & $1.17 \pm 0.04$ & $0.17 \pm 0.01$ & 1.96 \\
\hline Sample 19 & $0.07 \pm 0.00$ & $0.01 \pm 0.00$ & N.D. & N.D. & 0.08 \\
\hline Sample 20 & N.D. & N.D. & N.D. & N.D. & N.D. \\
\hline Sample 21 & $0.07 \pm 0.00$ & $0.01 \pm 0.00$ & N.D. & N.D. & 0.08 \\
\hline Sample 22 & $0.30 \pm 0.00$ & $0.32 \pm 0.01$ & $1.20 \pm 0.02$ & $0.18 \pm 0.00$ & 2.00 \\
\hline Sample 23 & $0.30 \pm 0.01$ & $0.31 \pm 0.02$ & $1.17 \pm 0.10$ & $0.17 \pm 0.01$ & 1.95 \\
\hline Sample 24 & $0.07 \pm 0.00$ & $0.01 \pm 0.00$ & N.D. & N.D. & 0.08 \\
\hline Sample 25 & N.D. & N.D. & N.D. & N.D. & N.D. \\
\hline Sample 26 & N.D. & N.D. & N.D. & N.D. & N.D. \\
\hline Sample 27 & $0.07 \pm 0.00$ & $0.01 \pm 0.00$ & N.D. & N.D. & 0.08 \\
\hline Sample 28 & N.D. & N.D. & N.D. & N.D. & N.D. \\
\hline Sample 29 & N.D. & N.D. & N.D. & N.D. & N.D. \\
\hline Sample 30 & $0.08 \pm 0.00$ & $0.02 \pm 0.00$ & N.D. & N.D. & 0.10 \\
\hline
\end{tabular}


Table 3. Cont.

\begin{tabular}{|c|c|c|c|c|c|}
\hline \multirow{2}{*}{ Sample } & \multicolumn{5}{|c|}{ Concentration $(\mu \mathrm{g} / \mathrm{kg})^{(1)}$} \\
\hline & $\mathrm{BaA}$ & CHR & $\mathrm{BbF}$ & BaP & PAH4 \\
\hline Sample 31 & $0.34 \pm 0.00$ & $0.38 \pm 0.00$ & $1.48 \pm 0.01$ & $0.21 \pm 0.00$ & 2.42 \\
\hline Sample 32 & $0.40 \pm 0.00$ & $0.46 \pm 0.01$ & $1.84 \pm 0.03$ & $0.27 \pm 0.00$ & 2.97 \\
\hline Sample 33 & $0.32 \pm 0.04$ & $0.35 \pm 0.05$ & $1.31 \pm 0.24$ & $0.19 \pm 0.03$ & 2.17 \\
\hline Sample 34 & $0.29 \pm 0.03$ & $0.31 \pm 0.03$ & $1.14 \pm 0.16$ & $0.17 \pm 0.02$ & 1.91 \\
\hline Sample 35 & $0.07 \pm 0.00$ & $0.01 \pm 0.00$ & N.D. & N.D. & 0.08 \\
\hline Sample 36 & $0.08 \pm 0.00$ & $0.02 \pm 0.00$ & N.D. & N.D. & 0.10 \\
\hline Sample 37 & $0.07 \pm 0.00$ & $0.01 \pm 0.00$ & N.D. & N.D. & 0.08 \\
\hline Sample 38 & $0.30 \pm 0.00$ & $0.32 \pm 0.00$ & $1.19 \pm 0.01$ & $0.17 \pm 0.00$ & 1.98 \\
\hline Sample 39 & $0.31 \pm 0.02$ & $0.33 \pm 0.03$ & $1.25 \pm 0.14$ & $0.18 \pm 0.02$ & 2.08 \\
\hline Sample 40 & $0.07 \pm 0.00$ & $0.01 \pm 0.00$ & N.D. & N.D. & 0.08 \\
\hline Sample 41 & $0.07 \pm 0.00$ & $0.01 \pm 0.00$ & N.D. & N.D. & 0.08 \\
\hline Sample 42 & $0.32 \pm 0.01$ & $0.35 \pm 0.01$ & $1.32 \pm 0.04$ & $0.19 \pm 0.00$ & 2.18 \\
\hline Sample 43 & N.D. & N.D. & N.D. & N.D. & N.D. \\
\hline Sample 44 & $0.36 \pm 0.05$ & $0.40 \pm 0.07$ & $1.57 \pm 0.35$ & $0.23 \pm 0.05$ & 2.56 \\
\hline Sample 45 & N.D. & N.D. & N.D. & N.D. & N.D. \\
\hline Sample 46 & $0.32 \pm 0.02$ & $0.34 \pm 0.03$ & $1.30 \pm 0.13$ & $0.19 \pm 0.02$ & 2.15 \\
\hline Sample 47 & $0.36 \pm 0.05$ & $0.40 \pm 0.07$ & $1.58 \pm 0.33$ & $0.23 \pm 0.05$ & 2.57 \\
\hline Sample 48 & $0.07 \pm 0.00$ & $0.01 \pm 0.00$ & N.D. & N.D. & 0.08 \\
\hline Sample 49 & N.D. & N.D. & N.D. & N.D. & N.D. \\
\hline Sample 50 & $0.07 \pm 0.00$ & $0.01 \pm 0.01$ & N.D. & N.D. & 0.09 \\
\hline
\end{tabular}

(1) Concentration values were expressed with mean \pm standard deviation. ${ }^{(2)}$ Concentration values below LOD were expressed as N.D. (not detected).

Table 4. PAH4 concentration in herbal medicines analyzed using QuEChERS pretreatment method.

\begin{tabular}{|c|c|c|c|c|c|}
\hline \multirow{2}{*}{ Sample } & \multicolumn{5}{|c|}{ Concentration $(\mu \mathrm{g} / \mathrm{kg})^{(1)}$} \\
\hline & $\mathrm{BaA}$ & CHR & $\mathrm{BbF}$ & BaP & PAH4 \\
\hline Sample 1 & N.D. ${ }^{(2)}$ & N.D. & $0.26 \pm 0.03$ & N.D. & 0.26 \\
\hline Sample 2 & N.D. & $0.05 \pm 0.00$ & $0.25 \pm 0.01$ & $0.04 \pm 0.00$ & 0.35 \\
\hline Sample 3 & N.D. & $0.05 \pm 0.00$ & $0.25 \pm 0.00$ & $0.04 \pm 0.00$ & 0.35 \\
\hline Sample 4 & $0.47 \pm 0.01$ & $0.64 \pm 0.01$ & $2.52 \pm 0.06$ & $0.38 \pm 0.01$ & 4.01 \\
\hline Sample 5 & N.D. & $0.05 \pm 0.00$ & $0.25 \pm 0.00$ & $0.04 \pm 0.00$ & 0.34 \\
\hline Sample 6 & $0.61 \pm 0.09$ & $0.80 \pm 0.10$ & $3.14 \pm 0.40$ & $0.47 \pm 0.06$ & 5.02 \\
\hline Sample 7 & $0.02 \pm 0.01$ & $0.15 \pm 0.01$ & $0.63 \pm 0.05$ & $0.10 \pm 0.01$ & 0.91 \\
\hline Sample 8 & $0.17 \pm 0.01$ & $0.32 \pm 0.01$ & $1.28 \pm 0.03$ & $0.19 \pm 0.00$ & 1.97 \\
\hline Sample 9 & N.D. & $0.11 \pm 0.00$ & $0.46 \pm 0.00$ & $0.07 \pm 0.00$ & 0.64 \\
\hline Sample 10 & $0.42 \pm 0.02$ & $0.59 \pm 0.02$ & $2.33 \pm 0.08$ & $0.35 \pm 0.01$ & 3.70 \\
\hline Sample 11 & $0.38 \pm 0.04$ & $0.55 \pm 0.04$ & $2.15 \pm 0.16$ & $0.32 \pm 0.02$ & 3.40 \\
\hline Sample 12 & N.D. & $0.06 \pm 0.00$ & $0.27 \pm 0.00$ & $0.05 \pm 0.00$ & 0.37 \\
\hline Sample 13 & N.D. & $0.05 \pm 0.00$ & $0.23 \pm 0.01$ & $0.04 \pm 0.00$ & 0.31 \\
\hline Sample 14 & N.D. & $0.05 \pm 0.00$ & $0.25 \pm 0.00$ & $0.04 \pm 0.00$ & 0.34 \\
\hline Sample 15 & $0.38 \pm 0.03$ & $0.55 \pm 0.03$ & $2.17 \pm 0.13$ & $0.33 \pm 0.02$ & 3.43 \\
\hline Sample 16 & N.D. & $0.04 \pm 0.00$ & $0.22 \pm 0.00$ & $0.04 \pm 0.00$ & 0.31 \\
\hline Sample 17 & $0.43 \pm 0.01$ & $0.60 \pm 0.01$ & $2.36 \pm 0.06$ & $0.35 \pm 0.01$ & 3.75 \\
\hline Sample 18 & $0.41 \pm 0.01$ & $0.58 \pm 0.01$ & $2.28 \pm 0.05$ & $0.34 \pm 0.01$ & 3.62 \\
\hline Sample 19 & N.D. & $0.05 \pm 0.00$ & $0.23 \pm 0.00$ & $0.04 \pm 0.00$ & 0.32 \\
\hline Sample 20 & N.D. & N.D. & $0.22 \pm 0.01$ & N.D. & 0.22 \\
\hline Sample 21 & N.D. & $0.05 \pm 0.00$ & $0.25 \pm 0.00$ & $0.04 \pm 0.00$ & 0.34 \\
\hline Sample 22 & $0.42 \pm 0.01$ & $0.59 \pm 0.01$ & $2.32 \pm 0.03$ & $0.35 \pm 0.01$ & 3.68 \\
\hline Sample 23 & $0.41 \pm 0.03$ & $0.58 \pm 0.03$ & $2.27 \pm 0.13$ & $0.34 \pm 0.02$ & 3.60 \\
\hline Sample 24 & N.D. & $0.04 \pm 0.00$ & $0.22 \pm 0.00$ & $0.04 \pm 0.00$ & 0.30 \\
\hline Sample 25 & N.D. & N.D. & $0.22 \pm 0.01$ & N.D. & 0.22 \\
\hline Sample 26 & N.D. & N.D. & $0.22 \pm 0.00$ & N.D. & 0.22 \\
\hline Sample 27 & N.D. & $0.05 \pm 0.00$ & $0.23 \pm 0.01$ & $0.04 \pm 0.00$ & 0.31 \\
\hline
\end{tabular}


Table 4. Cont.

\begin{tabular}{|c|c|c|c|c|c|}
\hline \multirow{2}{*}{ Sample } & \multicolumn{5}{|c|}{ Concentration $(\mu \mathrm{g} / \mathrm{kg})^{(1)}$} \\
\hline & $\mathrm{BaA}$ & CHR & $\mathrm{BbF}$ & BaP & PAH4 \\
\hline Sample 28 & N.D. & N.D. & $0.21 \pm 0.01$ & N.D. & 0.21 \\
\hline Sample 29 & N.D. & N.D. & $0.22 \pm 0.00$ & N.D. & 0.22 \\
\hline Sample 30 & N.D. & $0.07 \pm 0.00$ & $0.31 \pm 0.01$ & $0.05 \pm 0.00$ & 0.43 \\
\hline Sample 31 & $0.51 \pm 0.00$ & $0.69 \pm 0.00$ & $2.71 \pm 0.01$ & $0.41 \pm 0.00$ & 4.32 \\
\hline Sample 32 & $0.63 \pm 0.01$ & $0.82 \pm 0.01$ & $3.23 \pm 0.04$ & $0.48 \pm 0.01$ & 5.16 \\
\hline Sample 33 & $0.46 \pm 0.08$ & $0.63 \pm 0.09$ & $2.48 \pm 0.33$ & $0.37 \pm 0.05$ & 3.94 \\
\hline Sample 34 & $0.40 \pm 0.05$ & $0.57 \pm 0.06$ & $2.23 \pm 0.23$ & $0.34 \pm 0.03$ & 3.53 \\
\hline Sample 35 & N.D. & $0.05 \pm 0.00$ & $0.24 \pm 0.01$ & $0.04 \pm 0.00$ & 0.33 \\
\hline Sample 36 & N.D. & $0.07 \pm 0.01$ & $0.31 \pm 0.02$ & $0.05 \pm 0.00$ & 0.43 \\
\hline Sample 37 & N.D. & $0.05 \pm 0.00$ & $0.25 \pm 0.00$ & $0.04 \pm 0.00$ & 0.35 \\
\hline Sample 38 & $0.41 \pm 0.00$ & $0.58 \pm 0.00$ & $2.30 \pm 0.01$ & $0.35 \pm 0.00$ & 3.65 \\
\hline Sample 39 & $0.44 \pm 0.05$ & $0.61 \pm 0.05$ & $2.40 \pm 0.20$ & $0.36 \pm 0.03$ & 3.80 \\
\hline Sample 40 & N.D. & $0.05 \pm 0.00$ & $0.23 \pm 0.00$ & $0.04 \pm 0.00$ & 0.32 \\
\hline Sample 41 & N.D. & $0.05 \pm 0.00$ & $0.24 \pm 0.01$ & $0.04 \pm 0.00$ & 0.33 \\
\hline Sample 42 & $0.46 \pm 0.01$ & $0.63 \pm 0.01$ & $2.49 \pm 0.05$ & $0.37 \pm 0.01$ & 3.96 \\
\hline Sample 43 & N.D. & N.D. & $0.22 \pm 0.02$ & N.D. & 0.22 \\
\hline Sample 44 & $0.54 \pm 0.12$ & $0.72 \pm 0.13$ & $2.84 \pm 0.49$ & $0.42 \pm 0.07$ & 4.53 \\
\hline Sample 45 & N.D. & N.D. & $0.23 \pm 0.01$ & N.D. & 0.23 \\
\hline Sample 46 & $0.45 \pm 0.04$ & $0.63 \pm 0.05$ & $2.46 \pm 0.18$ & $0.37 \pm 0.03$ & 3.91 \\
\hline Sample 47 & $0.54 \pm 0.11$ & $0.73 \pm 0.12$ & $2.85 \pm 0.47$ & $0.43 \pm 0.07$ & 4.55 \\
\hline Sample 48 & N.D. & $0.04 \pm 0.01$ & $0.22 \pm 0.02$ & $0.04 \pm 0.00$ & 0.30 \\
\hline Sample 49 & N.D. & N.D. & N.D. & N.D. & N.D. \\
\hline Sample 50 & N.D. & $0.05 \pm 0.01$ & $0.25 \pm 0.04$ & $0.04 \pm 0.01$ & 0.35 \\
\hline
\end{tabular}

(1) Concentration values were expressed with mean \pm standard deviation. ${ }^{(2)}$ Concentration values below the LOD were expressed as N.D. (not detected).

\subsection{Exposure Assessment}

The average TEQ values for the $\mathrm{BaP}$ and $\mathrm{PAH} 4$ of herbal medicine ingredients were determined based on the TEF values $(0.10,0.01,0.10$, and 1.00 for BaA, CHR, BbF, and BaP, respectively) proposed by [13], as shown in Tables 5 and 6 . The TEQ values obtained using the conventional pretreatment method were $0.00-0.27 \mu \mathrm{g} / \mathrm{kg}$ for BaP and $0.00-0.50 \mu \mathrm{g} / \mathrm{kg}$ for PAH4. When using the QuEChERS method, the TEQ values for BaP and PAH4 were $0.00-0.48 \mu \mathrm{g} / \mathrm{kg}$ and $0.03-0.88 \mu \mathrm{g} / \mathrm{kg}$, respectively.

Table 5. TEQ values for PAH4 concentration in herbal medicines analyzed using typical pretreatment method.

\begin{tabular}{cccccc}
\hline Sample & \multicolumn{5}{c}{ TEQ Value $(\boldsymbol{\mu g} / \mathbf{k g})$} \\
\cline { 2 - 6 } & BaA & CHR & BbF & BaP & PAH4 \\
\hline TEF & 0.10 & 0.01 & 0.10 & 1.00 & N.D. \\
Sample 1 & N.D. ${ }^{(1)}$ & N.D. & N.D. & N.D. & $7.45 \times 10^{-3}$ \\
Sample 2 & $7.34 \times 10^{-3}$ & $1.10 \times 10^{-4}$ & N.D. & N.D. & $7.48 \times 10^{-3}$ \\
Sample 3 & $7.37 \times 10^{-3}$ & $1.14 \times 10^{-4}$ & N.D. & N.D. & $3.66 \times 10^{-1}$ \\
Sample 4 & $3.24 \times 10^{-2}$ & $3.52 \times 10^{-3}$ & $1.34 \times 10^{-1}$ & $1.96 \times 10^{-1}$ & $7.42 \times 10^{-3}$ \\
Sample 5 & $7.31 \times 10^{-3}$ & $1.07 \times 10^{-4}$ & N.D. & N.D. & $4.78 \times 10^{-1}$ \\
Sample 6 & $3.91 \times 10^{-2}$ & $4.44 \times 10^{-3}$ & $1.78 \times 10^{-1}$ & $2.57 \times 10^{-1}$ & $2.14 \times 10^{-2}$ \\
Sample 7 & $1.16 \times 10^{-2}$ & $6.84 \times 10^{-4}$ & N.D. & $9.17 \times 10^{-3}$ & $1.39 \times 10^{-1}$ \\
Sample 8 & $1.87 \times 10^{-2}$ & $1.65 \times 10^{-3}$ & $4.58 \times 10^{-2}$ & $7.32 \times 10^{-2}$ & $1.01 \times 10^{-1}$ \\
Sample 9 & $9.69 \times 10^{-3}$ & $4.29 \times 10^{-4}$ & N.D. & N.D. & $3.32 \times 10^{-1}$ \\
Sample 10 & $3.03 \times 10^{-2}$ & $3.23 \times 10^{-3}$ & $1.21 \times 10^{-1}$ & $1.77 \times 10^{-1}$ & $2.99 \times 10^{-1}$ \\
Sample 11 & $2.83 \times 10^{-2}$ & $2.96 \times 10^{-3}$ & $1.08 \times 10^{-1}$ & $1.60 \times 10^{-1}$ & $7.70 \times 10^{-3}$ \\
Sample 12 & $7.56 \times 10^{-3}$ & $1.40 \times 10^{-4}$ & N.D. & N.D. & $7.16 \times 10^{-3}$ \\
Sample 13 & $7.08 \times 10^{-3}$ & $7.54 \times 10^{-5}$ & N.D. & N.D. &
\end{tabular}


Table 5. Cont.

\begin{tabular}{|c|c|c|c|c|c|}
\hline \multirow{2}{*}{ Sample } & \multicolumn{5}{|c|}{ TEQ Value $(\mu \mathrm{g} / \mathrm{kg})$} \\
\hline & $\mathrm{BaA}$ & CHR & $\mathrm{BbF}$ & BaP & PAH4 \\
\hline Sample 14 & $7.31 \times 10^{-3}$ & $1.06 \times 10^{-4}$ & N.D. & N.D. & $7.42 \times 10^{-3}$ \\
\hline Sample 15 & $2.85 \times 10^{-2}$ & $2.99 \times 10^{-3}$ & $1.09 \times 10^{-1}$ & $1.61 \times 10^{-1}$ & $3.02 \times 10^{-1}$ \\
\hline Sample 16 & $7.03 \times 10^{-3}$ & $6.86 \times 10^{-5}$ & N.D. & N.D. & $7.10 \times 10^{-3}$ \\
\hline Sample 17 & $3.06 \times 10^{-2}$ & $3.28 \times 10^{-3}$ & $1.23 \times 10^{-1}$ & $1.80 \times 10^{-1}$ & $3.37 \times 10^{-1}$ \\
\hline Sample 18 & $2.98 \times 10^{-2}$ & $3.16 \times 10^{-3}$ & $1.17 \times 10^{-1}$ & $1.72 \times 10^{-1}$ & $3.23 \times 10^{-1}$ \\
\hline Sample 19 & $7.16 \times 10^{-3}$ & $8.50 \times 10^{-5}$ & N.D. & N.D. & $7.24 \times 10^{-3}$ \\
\hline Sample 20 & N.D. & N.D. & N.D. & N.D. & N.D. \\
\hline Sample 21 & $7.28 \times 10^{-3}$ & $1.02 \times 10^{-4}$ & N.D. & N.D. & $7.38 \times 10^{-3}$ \\
\hline Sample 22 & $3.02 \times 10^{-2}$ & $3.22 \times 10^{-3}$ & $1.20 \times 10^{-1}$ & $1.76 \times 10^{-1}$ & $3.30 \times 10^{-1}$ \\
\hline Sample 23 & $2.96 \times 10^{-2}$ & $3.14 \times 10^{-3}$ & $1.17 \times 10^{-1}$ & $1.71 \times 10^{-1}$ & $3.21 \times 10^{-1}$ \\
\hline Sample 24 & $6.99 \times 10^{-3}$ & $6.21 \times 10^{-5}$ & N.D. & N.D. & $7.05 \times 10^{-3}$ \\
\hline Sample 25 & N.D. & N.D. & N.D. & N.D. & N.D. \\
\hline Sample 26 & N.D. & N.D. & N.D. & N.D. & N.D. \\
\hline Sample 27 & $7.08 \times 10^{-3}$ & $7.51 \times 10^{-5}$ & N.D. & N.D. & $7.16 \times 10^{-3}$ \\
\hline Sample 28 & N.D. & N.D. & N.D. & N.D. & N.D. \\
\hline Sample 29 & N.D. & N.D. & N.D. & N.D. & N.D. \\
\hline Sample 30 & $8.01 \times 10^{-3}$ & $2.02 \times 10^{-4}$ & N.D. & N.D. & $8.22 \times 10^{-3}$ \\
\hline Sample 31 & $3.45 \times 10^{-2}$ & $3.80 \times 10^{-3}$ & $1.48 \times 10^{-1}$ & $2.15 \times 10^{-1}$ & $4.01 \times 10^{-1}$ \\
\hline Sample 32 & $4.01 \times 10^{-2}$ & $4.57 \times 10^{-3}$ & $1.84 \times 10^{-1}$ & $2.66 \times 10^{-1}$ & $4.95 \times 10^{-1}$ \\
\hline Sample 33 & $3.19 \times 10^{-2}$ & $3.67 \times 10^{-3}$ & $1.31 \times 10^{-1}$ & $1.92 \times 10^{-1}$ & $3.59 \times 10^{-1}$ \\
\hline Sample 34 & $2.92 \times 10^{-2}$ & $3.09 \times 10^{-3}$ & $1.14 \times 10^{-1}$ & $1.68 \times 10^{-1}$ & $3.14 \times 10^{-1}$ \\
\hline Sample 35 & $7.21 \times 10^{-3}$ & $9.20 \times 10^{-5}$ & N.D. & N.D. & $7.30 \times 10^{-3}$ \\
\hline Sample 36 & $8.02 \times 10^{-3}$ & $2.03 \times 10^{-4}$ & N.D. & N.D. & $8.23 \times 10^{-3}$ \\
\hline Sample 37 & $7.34 \times 10^{-3}$ & $1.10 \times 10^{-4}$ & N.D. & N.D. & $7.45 \times 10^{-3}$ \\
\hline Sample 38 & $2.99 \times 10^{-2}$ & $3.19 \times 10^{-3}$ & $1.19 \times 10^{-1}$ & $1.74 \times 10^{-1}$ & $3.26 \times 10^{-1}$ \\
\hline Sample 39 & $3.10 \times 10^{-2}$ & $3.33 \times 10^{-3}$ & $1.25 \times 10^{-1}$ & $1.84 \times 10^{-1}$ & $3.44 \times 10^{-1}$ \\
\hline Sample 40 & $7.13 \times 10^{-3}$ & $8.15 \times 10^{-5}$ & N.D. & N.D. & $7.21 \times 10^{-3}$ \\
\hline Sample 41 & $7.25 \times 10^{-3}$ & $9.80 \times 10^{-5}$ & N.D. & N.D. & $7.35 \times 10^{-3}$ \\
\hline Sample 42 & $3.21 \times 10^{-2}$ & $3.48 \times 10^{-3}$ & $1.32 \times 10^{-1}$ & $1.93 \times 10^{-1}$ & $3.61 \times 10^{-1}$ \\
\hline Sample 43 & N.D. & N.D. & N.D. & N.D. & N.D. \\
\hline Sample 44 & $3.59 \times 10^{-2}$ & $4.00 \times 10^{-3}$ & $1.57 \times 10^{-1}$ & $2.28 \times 10^{-1}$ & $4.25 \times 10^{-1}$ \\
\hline Sample 45 & N.D. & N.D. & N.D. & N.D. & N.D. \\
\hline Sample 46 & $3.17 \times 10^{-2}$ & $3.43 \times 10^{-3}$ & $1.30 \times 10^{-1}$ & $1.90 \times 10^{-1}$ & $3.56 \times 10^{-1}$ \\
\hline Sample 47 & $3.60 \times 10^{-2}$ & $4.01 \times 10^{-3}$ & $1.58 \times 10^{-1}$ & $2.29 \times 10^{-1}$ & $4.27 \times 10^{-1}$ \\
\hline Sample 48 & $7.01 \times 10^{-3}$ & $6.54 \times 10^{-5}$ & N.D. & N.D. & $7.08 \times 10^{-3}$ \\
\hline Sample 49 & N.D. & N.D. & N.D. & N.D. & N.D. \\
\hline Sample 50 & $7.38 \times 10^{-3}$ & $1.15 \times 10^{-4}$ & N.D. & N.D. & $7.49 \times 10^{-3}$ \\
\hline
\end{tabular}

(1) Concentration values below the LOD were expressed as N.D. (not detected).

Table 6. TEQ values for PAH4 concentration in herbal medicines analyzed using QuEChERS pretreatment method.

\begin{tabular}{|c|c|c|c|c|c|}
\hline \multirow{2}{*}{ Sample } & \multicolumn{5}{|c|}{ TEQ Value ( $\mu \mathrm{g} / \mathrm{kg})$} \\
\hline & $\mathbf{B a A}$ & CHR & $\mathrm{BbF}$ & BaP & PAH4 \\
\hline TEF & 0.10 & 0.01 & 0.10 & 1.00 & \\
\hline Sample 1 & N.D. ${ }^{(1)}$ & N.D. & $2.58 \times 10^{-2}$ & N.D. & $2.58 \times 10^{-2}$ \\
\hline Sample 2 & N.D. & $5.17 \times 10^{-4}$ & $2.51 \times 10^{-2}$ & $4.25 \times 10^{-2}$ & $6.82 \times 10^{-2}$ \\
\hline Sample 3 & N.D. & $5.24 \times 10^{-4}$ & $2.54 \times 10^{-2}$ & $4.29 \times 10^{-2}$ & $6.88 \times 10^{-2}$ \\
\hline Sample 4 & $4.65 \times 10^{-2}$ & $6.42 \times 10^{-3}$ & $2.52 \times 10^{-1}$ & $3.78 \times 10^{-1}$ & $6.83 \times 10^{-1}$ \\
\hline Sample 5 & N.D. & $5.12 \times 10^{-4}$ & $2.49 \times 10^{-2}$ & $4.22 \times 10^{-2}$ & $6.76 \times 10^{-2}$ \\
\hline Sample 6 & $6.10 \times 10^{-2}$ & $8.01 \times 10^{-3}$ & $3.14 \times 10^{-1}$ & $4.68 \times 10^{-1}$ & $8.51 \times 10^{-1}$ \\
\hline Sample 7 & $2.18 \times 10^{-3}$ & $1.51 \times 10^{-3}$ & $6.33 \times 10^{-2}$ & $9.89 \times 10^{-2}$ & $1.66 \times 10^{-1}$ \\
\hline Sample 8 & $1.74 \times 10^{-2}$ & $3.19 \times 10^{-3}$ & $1.28 \times 10^{-1}$ & $1.94 \times 10^{-1}$ & $3.43 \times 10^{-1}$ \\
\hline Sample 9 & N.D. & $1.07 \times 10^{-3}$ & $4.64 \times 10^{-2}$ & $7.39 \times 10^{-2}$ & $1.21 \times 10^{-1}$ \\
\hline
\end{tabular}


Table 6. Cont.

\begin{tabular}{|c|c|c|c|c|c|}
\hline \multirow{2}{*}{ Sample } & \multicolumn{5}{|c|}{ TEQ Value $(\mu \mathrm{g} / \mathrm{kg})$} \\
\hline & $\mathrm{BaA}$ & CHR & $\mathrm{BbF}$ & BaP & PAH4 \\
\hline Sample 10 & $4.21 \times 10^{-2}$ & $5.92 \times 10^{-3}$ & $2.33 \times 10^{-1}$ & $3.50 \times 10^{-1}$ & $6.31 \times 10^{-1}$ \\
\hline Sample 11 & $3.79 \times 10^{-2}$ & $5.46 \times 10^{-3}$ & $2.15 \times 10^{-1}$ & $3.23 \times 10^{-1}$ & $5.82 \times 10^{-1}$ \\
\hline Sample 12 & N.D. & $5.69 \times 10^{-4}$ & $2.71 \times 10^{-2}$ & $4.54 \times 10^{-2}$ & $7.31 \times 10^{-2}$ \\
\hline Sample 13 & N.D. & $4.57 \times 10^{-4}$ & $2.28 \times 10^{-2}$ & $3.91 \times 10^{-2}$ & $6.24 \times 10^{-2}$ \\
\hline Sample 14 & N.D. & $5.11 \times 10^{-4}$ & $2.49 \times 10^{-2}$ & $4.22 \times 10^{-2}$ & $6.76 \times 10^{-2}$ \\
\hline Sample 15 & $3.83 \times 10^{-2}$ & $5.51 \times 10^{-3}$ & $2.17 \times 10^{-1}$ & $3.26 \times 10^{-1}$ & $5.87 \times 10^{-1}$ \\
\hline Sample 16 & N.D. & $4.46 \times 10^{-4}$ & $2.23 \times 10^{-2}$ & $3.84 \times 10^{-2}$ & $6.12 \times 10^{-2}$ \\
\hline Sample 17 & $4.28 \times 10^{-2}$ & $6.00 \times 10^{-3}$ & $2.36 \times 10^{-1}$ & $3.54 \times 10^{-1}$ & $6.39 \times 10^{-1}$ \\
\hline Sample 18 & $4.09 \times 10^{-2}$ & $5.80 \times 10^{-3}$ & $2.28 \times 10^{-1}$ & $3.43 \times 10^{-2}$ & $6.18 \times 10^{-1}$ \\
\hline Sample 19 & N.D. & $4.74 \times 10^{-4}$ & $2.34 \times 10^{-2}$ & $4.01 \times 10^{-2}$ & $6.40 \times 10^{-2}$ \\
\hline Sample 20 & N.D. & N.D. & $2.17 \times 10^{-2}$ & N.D. & $2.17 \times 10^{-2}$ \\
\hline Sample 21 & N.D. & $5.04 \times 10^{-4}$ & $2.46 \times 10^{-2}$ & $4.18 \times 10^{-2}$ & $6.69 \times 10^{-2}$ \\
\hline Sample 22 & $4.19 \times 10^{-2}$ & $5.90 \times 10^{-3}$ & $2.32 \times 10^{-1}$ & $3.48 \times 10^{-1}$ & $6.28 \times 10^{-1}$ \\
\hline Sample 23 & $4.07 \times 10^{-2}$ & $5.77 \times 10^{-3}$ & $2.27 \times 10^{-1}$ & $3.41 \times 10^{-1}$ & $6.15 \times 10^{-1}$ \\
\hline Sample 24 & N.D. & $4.34 \times 10^{-4}$ & $2.19 \times 10^{-2}$ & $3.78 \times 10^{-2}$ & $6.02 \times 10^{-2}$ \\
\hline Sample 25 & N.D. & N.D. & $2.24 \times 10^{-2}$ & N.D. & $2.24 \times 10^{-2}$ \\
\hline Sample 26 & N.D. & N.D. & $2.22 \times 10^{-2}$ & N.D. & $2.22 \times 10^{-2}$ \\
\hline Sample 27 & N.D. & $4.57 \times 10^{-4}$ & $2.28 \times 10^{-2}$ & $3.91 \times 10^{-2}$ & $6.23 \times 10^{-2}$ \\
\hline Sample 28 & N.D. & N.D. & $2.14 \times 10^{-2}$ & N.D. & $2.14 \times 10^{-2}$ \\
\hline Sample 29 & N.D. & N.D. & $2.19 \times 10^{-2}$ & N.D. & $2.19 \times 10^{-2}$ \\
\hline Sample 30 & N.D. & $6.77 \times 10^{-4}$ & $3.12 \times 10^{-2}$ & $5.16 \times 10^{-2}$ & $8.35 \times 10^{-2}$ \\
\hline Sample 31 & $5.10 \times 10^{-2}$ & $6.90 \times 10^{-3}$ & $2.17 \times 10^{-1}$ & $4.05 \times 10^{-1}$ & $7.34 \times 10^{-1}$ \\
\hline Sample 32 & $6.31 \times 10^{-2}$ & $8.24 \times 10^{-3}$ & $3.23 \times 10^{-1}$ & $4.81 \times 10^{-1}$ & $8.75 \times 10^{-1}$ \\
\hline Sample 33 & $4.56 \times 10^{-2}$ & $6.31 \times 10^{-3}$ & $2.48 \times 10^{-1}$ & $3.72 \times 10^{-1}$ & $6.72 \times 10^{-1}$ \\
\hline Sample 34 & $3.98 \times 10^{-2}$ & $5.67 \times 10^{-3}$ & $2.23 \times 10^{-1}$ & $3.35 \times 10^{-1}$ & $6.04 \times 10^{-1}$ \\
\hline Sample 35 & N.D. & $4.86 \times 10^{-4}$ & $2.39 \times 10^{-2}$ & $4.07 \times 10^{-2}$ & $6.51 \times 10^{-2}$ \\
\hline Sample 36 & N.D. & $6.79 \times 10^{-4}$ & $3.13 \times 10^{-2}$ & $5.17 \times 10^{-2}$ & $8.37 \times 10^{-2}$ \\
\hline Sample 37 & N.D. & $5.18 \times 10^{-4}$ & $2.51 \times 10^{-2}$ & $4.25 \times 10^{-2}$ & $6.82 \times 10^{-2}$ \\
\hline Sample 38 & $4.14 \times 10^{-2}$ & $5.84 \times 10^{-3}$ & $2.30 \times 10^{-1}$ & $3.45 \times 10^{-1}$ & $6.23 \times 10^{-1}$ \\
\hline Sample 39 & $4.36 \times 10^{-2}$ & $6.09 \times 10^{-3}$ & $2.40 \times 10^{-1}$ & $3.59 \times 10^{-1}$ & $6.49 \times 10^{-1}$ \\
\hline Sample 40 & N.D. & $4.68 \times 10^{-4}$ & $2.32 \times 10^{-2}$ & $3.97 \times 10^{-2}$ & $6.34 \times 10^{-2}$ \\
\hline Sample 41 & N.D. & $4.97 \times 10^{-4}$ & $2.43 \times 10^{-2}$ & $4.13 \times 10^{-2}$ & $6.61 \times 10^{-2}$ \\
\hline Sample 42 & $4.59 \times 10^{-2}$ & $6.34 \times 10^{-3}$ & $2.49 \times 10^{-1}$ & $3.74 \times 10^{-1}$ & $6.75 \times 10^{-1}$ \\
\hline Sample 43 & N.D. & N.D. & $2.19 \times 10^{-2}$ & N.D. & $2.19 \times 10^{-2}$ \\
\hline Sample 44 & $5.40 \times 10^{-2}$ & $7.24 \times 10^{-3}$ & $2.84 \times 10^{-1}$ & $4.25 \times 10^{-1}$ & $7.70 \times 10^{-1}$ \\
\hline Sample 45 & N.D. & N.D. & $2.26 \times 10^{-2}$ & N.D. & $2.26 \times 10^{-2}$ \\
\hline Sample 46 & $4.52 \times 10^{-2}$ & $6.26 \times 10^{-3}$ & $2.46 \times 10^{-1}$ & $3.69 \times 10^{-1}$ & $6.67 \times 10^{-1}$ \\
\hline Sample 47 & $5.43 \times 10^{-2}$ & $7.27 \times 10^{-3}$ & $2.85 \times 10^{-1}$ & $4.26 \times 10^{-1}$ & $7.73 \times 10^{-1}$ \\
\hline Sample 48 & N.D. & $4.40 \times 10^{-4}$ & $2.21 \times 10^{-2}$ & $3.81 \times 10^{-2}$ & $6.07 \times 10^{-2}$ \\
\hline Sample 49 & N.D. & N.D. & N.D. & N.D. & N.D. \\
\hline Sample 50 & N.D. & $5.26 \times 10^{-4}$ & $2.55 \times 10^{-2}$ & $4.30 \times 10^{-2}$ & $6.90 \times 10^{-2}$ \\
\hline
\end{tabular}

(1) Concentration values below the LOD were expressed as N.D. (not detected).

The DAI values of the samples were also calculated using each of the derived TEQ values (Table 7). As indicated in Equation (2) and mentioned in Section 2.10, DAI values are proportional to the TEQ value. The values obtained using the conventional pretreatment method were 0-1.42 $\times 10^{-8} \mu \mathrm{g} / \mathrm{kg} /$ day for BaP and $0-2.64 \times 10^{-8} \mu \mathrm{g} / \mathrm{kg} /$ day for PAH4 When using the QuEChERS method, the values were $0-2.57 \times 10^{-8} \mu \mathrm{g} / \mathrm{kg} /$ day for BaP and $1.38 \times 10^{-9}-4.67 \times 10^{-8} \mu \mathrm{g} / \mathrm{kg} /$ day for PAH4 . 
Table 7. Average DAI and MOE values in herbal medicine products.

\begin{tabular}{cccc}
\hline Treatment type & $\begin{array}{c}\text { DAI of BaP } \\
(\boldsymbol{\mu g} / \mathbf{k g} / \mathbf{d a y})\end{array}$ & $\begin{array}{c}\text { DAI of PAH4 } \\
(\boldsymbol{\mu g} / \mathbf{k g} / \text { day })\end{array}$ & MOE \\
\hline Typical & $4.02 \times 10^{-9}$ & $7.69 \times 10^{-9}$ & $1.30 \times 10^{10}$ \\
QuEChERS & $8.85 \times 10^{-9}$ & $1.60 \times 10^{-8}$ & $6.26 \times 10^{9}$ \\
\hline
\end{tabular}

\subsection{Risk Characterization}

Based on the data calculated through the exposure assessment, population-wide MOE values for the herbal medicine ingredients were obtained using the BMDL (100 $\mu \mathrm{g} / \mathrm{kg} \cdot \mathrm{BW} /$ day) and the dietary exposure. The MOE values for the conventional and QuEChERS methods were $1.30 \times 10^{10}$ and $6.26 \times 10^{9}$, respectively. These two values were $>1,000,000$, thus indicating a "negligible concern." The excessive cancer risk values of PAH4 for the total population obtained using the conventional and QuEChERS pretreatment methods were $5.61 \times 10^{-11}$ and $1.17 \times 10^{-10}$, respectively, and therefore the PAH4 concentration level of the herbal medicine ingredient was deemed "safe and acceptable."

\section{Discussion}

To improve the recovery of the QuEChERS method, selecting an appropriate extraction solvent with a similar polarity to that of the 4 PAHs is key. Most related studies reported that acetonitrile, acetone, and ethyl acetate were suitable extraction solvents because they had an appropriate polarity for most compounds and rendered good recovery rates [15]. In some cases, $1 \%$ acetic acid was added to the acetonitrile solution to improve recovery $[16,17]$. However, more recent studies reported that using hexane:acetone $(1: 1)$ as the extraction solvent instead of acetonitrile results in higher PAH recovery rates, and is therefore widely used for the determination of pesticides as an extraction solvent in the QuEChERS method [18]. Acetone is mixed with hexane to induce a distinct separation from the water phase, as hexane dissolves nonpolar molecules [15]. Thus, this solvent mixture is mainly used for the extraction of nonpolar compounds and is therefore suitable for the determination of the four PAHs examined herein, which are nonpolar molecules [19]. Furthermore, a $1 \mathrm{~h}$ hydration step is implemented before applying the extraction solvent to the sample to facilitate proper partitioning $[15,20]$. For the silica SPE clean-up step, larger molecular weight compounds required a stronger solvent to elute them from the silica. Therefore, $15 \%$ methylene chloride in hexane was employed for the 4 PAHs, which are considered mid-sized molecules. In this sense, the choice of solvent for 4PAH analysis is an important factor to optimize recovery rates. According to the European Commission (EC) Regulation No. 836/2011, the criteria for analyzing PAH4 (BaA, CHR, BbF, and $\mathrm{BaP}$ ) are $\mathrm{LOD} \leq 0.30 \mu \mathrm{g} / \mathrm{kg}, \mathrm{LOQ} \leq 0.90 \mu \mathrm{g} / \mathrm{kg}$, and recovery values of $50-120 \%$ [21]. [22] validated a method for PAH determination in wastewater and sediments and achieved an $\mathrm{R}^{2}>0.99$, $0.02-0.51 \mu \mathrm{g} / \mathrm{kg}$ LOD, $0.05-1.71 \mu \mathrm{g} / \mathrm{kg}$ LOQ, and 80-104\% recovery. [23] also validated a method to determine PAH8 in ready-to-eat food products. The correlation coefficients $\left(\mathrm{R}^{2}\right)$ were higher than 0.99 , the LOD and LOQ were $0.12-0.19 \mu \mathrm{g} / \mathrm{kg}$ and $0.36-0.57 \mu \mathrm{g} / \mathrm{kg}$, respectively, and the recovery and precision were $82.4-113.6 \%$ and $0.6-12.4 \%$ for interday analysis and $81.2-113.7 \%$ and $1.7-13.1 \%$ for intraday analysis, respectively. Therefore, the validation parameter values indicated that GC/MS and HPLC-FLD were suitable for the determination of PAH4 in herbal medicine ingredients.

The main purpose of this study was to determine whether the QuEChERS coupled with HPLC-FLD could replace the conventional pretreatment method coupled with GC/MS for the determination of PAH4 in herbal medicine ingredients. No substantial differences in sample concentration and recoveries were observed between the two methods. Nevertheless, the QuEChERS method was better suited for the detection of $\mathrm{BaP}$ and PAH4 concentrations. Few studies employed this method for the detection of PAHs in herbal medicine ingredients; however, some studies used this approach to detect residual pesticides in herbs or plants. Ref. [24] developed a QuEChERS-based method for the detection 
and quantification of pesticides in herbs and achieved recovery rates of $78.4-119.2 \%$ and relative standard deviations below $9.5 \%$. Using this method, the authors determined that each sample contained at least one of the examined pesticides. Other studies also applied QuEChERS to dried herbs and plants to detect pesticides and achieved $70 \%-120 \%$ recovery rates $[25,26]$. The authors thus concluded that this method could be used to monitor pesticides in herbs and plants. Due to the lack of information regarding the application of QuEChERS methods for the assessment of herbal medicine ingredients, assessing the viability of this approach to substitute the conventional pretreatment method was critical. Our results indicated that both the conventional and QuEChERS methods rendered similar recovery ranges and PAH4 detection concentrations. Therefore, we concluded that the QuEChERS method is an effective approach that could potentially replace the conventional PAH4 pretreatment method for the analysis of herbal medicine ingredients used in Korea. However, herbal medicine ingredients contain large amounts of coextractives, and therefore, further studies are required to assess the performance of the modified QuEChERS pretreatment method. Sadowska-Rociek et al. [8] applied the QuEChERS method to analyze black, green, red, and white tea. The authors mentioned that some modifications had to be made to ensure the successful determination of PAHs, as tea contains a variety of interfering substances such as caffeine, polyphenols, and chlorophyll, all of which impede accurate PAH determination.

The specific structures of the herbal medicine ingredients used in this study (i.e., roots, stems, flowers, fruits, seeds, leaves, or bark) were the same as those reported by [27]. PAH4 are often formed in herbal medicine ingredients during thermal processes such as roasting, smoking, or drying. In a study by [9], PAHs ranged from 6.5 to $1112.1 \mathrm{ng} / \mathrm{g}$ in tea products and crude herbal medicine ingredients. Further, Ref. [28] reported PAHs of $0.2-11.9 \mu \mathrm{g} / \mathrm{kg}$ in Chinese medicinal herbs. In Korea, the BaP and PAH4 concentration limits in herbal medicine ingredients are 5.0 and $10.0 \mu \mathrm{g} / \mathrm{kg}$, respectively. All 50 samples evaluated in this study exhibited BaP and PAH4 levels that were below the aforementioned guidelines when using both pretreatment methods and were thus considered safe. Similarly, [29] analyzed 93 herbal pills in Seoul, Korea, and reported that the PAH concentration of all of the samples was below $10 \mu \mathrm{g} / \mathrm{kg}$. Therefore, the PAH levels in herbal medicine ingredients used in Korea were deemed safe. Based on exposure assessment and cancer risk characterization, our study confirmed that the levels of PAH4 in herbal medicine ingredients were within safe and acceptable limits. Our findings were consistent with those reported by other studies $[29,30]$, which confirmed that tea leaves and herbal pills, respectively, contained safe PAH levels.

\section{Conclusions}

This study evaluated whether the QuEChERS pretreatment method coupled with HPLC-FLD could replace the conventional pretreatment method coupled with GC/MS for the determination of PAH4 (BaA, $\mathrm{CHR}, \mathrm{BbF}$, and $\mathrm{BaP})$ in herbal medicine ingredients. Both methods exhibited largely similar BaP and PAH4 detection performances. Further, the results of both pretreatment methods were used to evaluate the health risks associated with $\mathrm{BaP}$ and PAH4 in 50 herbal medicine ingredients. Based on European Union and Koreas toxicity guidelines, the $\mathrm{BaP}$ and PAH4 concentrations in all samples were deemed safe. Through exposure assessment and cancer risk characterization, the PAH4 levels in various herbal medicine ingredients were found to be within safe and acceptable limits. Taken together, our findings confirm that the QuEChERS method could effectively replace the conventional pretreatment method, thus providing a more practical means for the detection of PAHs in herbal medicine ingredients.

Supplementary Materials: The following are available online at https: / www.mdpi.com/article / 10.3390/foods10092200/s1, Figure S1: GC-MS chromatograms of PAH4 standards (A); PAH4 with spiked sample (B); two internal standards with blank sample (C), and chromatograms of PAHs for sample (D). Figure S2: HPLC chromatograms of four PAHs standards (A); four PAHs with spiked sample (B); internal standards with blank sample (C), and chromatograms of PAHs for sample (D). 
Author Contributions: H.-J.H.: formal analysis, writing—original draft preparation, data curation; S.-H.L.: conceptualization, software, visualization; Y.-Y.K.: investigation, validation; H.-S.S.: project administration, writing-review and editing. All authors have read and agreed to the published version of the manuscript.

Funding: This work was supported by Korea Environmental Industry \& Technology Institute (A11700197-0703-0).

Institutional Review Board Statement: Not applicable.

Informed Consent Statement: Not applicable.

Data Availability Statement: The datasets generated for this study are available on request to the corresponding author.

Conflicts of Interest: The authors declare no conflict of interest.

\section{References}

1. Ravindra, K.; Sokhi, R.; Van Grieken, R. Atmospheric polycyclic aromatic hydrocarbons: Source attribution, emission factors and regulation. Atmos. Environ. 2008, 42, 2895-2921. [CrossRef]

2. Moret, S.; Conte, L.S. Polycyclic aromatic hydrocarbons in edible fats and oils: Occurrence and analytical methods. J. Chromatogr. A 2000, 882, 245-253. [CrossRef]

3. Nikolaou, K.; Masclet, P.; Mouvier, G. PAH stability scale established in situ in an urban region. Sci. Total Environ. 1984, 36, 383-388. [CrossRef]

4. Arey, J.; Atkinson, R. Photochemical reactions of PAHs in the atmosphere. In PAHs. An Ecotoxicological Perspective, 1st ed.; Douben, P.E.T., Ed.; John Wiley \& Sons: Hoboken, NJ, USA, 2003; pp. 47-63.

5. IARC Monographs on the Evaluation of Carcinogenic Risks to Humans. Vol. 92, Some Non-Heterocyclic Polycyclic Aromatic Hydrocarbons and Some Related Exposures. 2010. Available online: http://monographs.iarc.fr/ENG/Monographs/vol92/ mono92.pdf (accessed on 10 September 2020).

6. Korea Food \& Drug Administration. Criteria and Method of Benzopyrene for Herbal; Notice No. 2009; Korea Food \& Drug Administration: Osong, Korea, 2009.

7. Ekner, H.; Dreij, K.; Sadiktsis, I. Determination of polycyclic aromatic hydrocarbons in commercial olive oils by HPLC/GC/MSOccurrence, composition and sources. Food Control 2021, 132, 108528. [CrossRef]

8. Sadowska-Rociek, A.; Surma, M.; Cieślik, E. Comparison of different modifications on QuEChERS sample preparation method for PAHs determination in black, green, red and white tea. Environ. Sci. Pollut. Res. 2014, 21, 1326-1338. [CrossRef]

9. Ishizaki, A.; Sito, K.; Kataoka, H. Analysis of contaminant polycyclic aromatic hydrocarbons in tea products and crude drugs. Anal. Methods 2011, 3, 299-305. [CrossRef] [PubMed]

10. Anastassiades, M.; Lehotay, S.J.; Štajnbaher, D.; Schenck, F.J. Fast and easy multiresidue method employing acetonitrile extraction/partitioning and "dispersive solid-phase extraction" for the determination of pesticide residues in produce. J. AOAC Int. 2003, 86, 412-431. [CrossRef] [PubMed]

11. Furlani, R.P.; Dias, F.F.; Nogueira, P.M.; Gomes, F.M.; Tfouni, S.A.; Camargo, M.C. Occurrence of macrocyclic lactones in milk and yogurt from Brazilian market. Food Control 2015, 48, 43-47. [CrossRef]

12. Tfouni, S.A.; Reis, R.M.; Kamikata, K.; Gomes, F.M.; Morgano, M.A.; Furlani, R.P. Polycyclic aromatic hydrocarbons in teas using QuEChERS and HPLC-FLD. Food Addit. Contam. Part B 2018, 11, 146-152. [CrossRef]

13. Nisbet, I.C.; Lagoy, P.K. Toxic equivalency factors (TEFs) for polycyclic aromatic hydrocarbons (PAHs). Regul. Toxicol. Pharm. 1992, 16, 290-300. [CrossRef]

14. Chen, C.; Chu, M. Dose-Response Analysis of Ingested Benzo (a) Pyrene (CAS No. 50-32-8); No. PB-93-167484/XAB; EPA-600/R92/045; Environmental Protection Agency: Washington, DC, USA, 1991.

15. Maštovská, K.; Lehotay, S.J. Evaluation of common organic solvents for gas chromatographic analysis and stability of multiclass pesticide residues. J. Chromatogr. A 2004, 1040, 259-272. [CrossRef] [PubMed]

16. Da Costa Morais, E.H.; Collins, C.H.; Jardim, I.C.S.F. Pesticide determination in sweet peppers using QuEChERS and LC-MS/MS. Food Chem. 2018, 249, 77-83. [CrossRef]

17. Lehotay, S.J.; Son, K.A.; Kwon, H.; Koesukwiwat, U.; Fu, W.; Mastovska, K.; Hoh, E.; Leepipatpiboon, N. Comparison of QuEChERS sample preparation methods for the analysis of pesticide residues in fruits and vegetables. J. Chromatogr. A 2010, 1217, 2548-2560. [CrossRef]

18. Julie, K.; Amanda, R.; Jack, C. Analytical Method of Polycyclis Aromatic Hydrocarbons(PAHs) in Yerba Mate Tea Using Modified QuEChERS, Solid Phase Extraction and GC-TOFMS and GC-MS/MS. 2017. Available online: https: //www.restek.com/fr/technical-literature-library/articles/analytical-method-for-polycyclic-aromatic-hydrocarbons-PAHSin-yerba-mate-tea-using-modified-quechers-solid-phase-extraction-and-GC-TOFMS-and-GC-MS-MS/ (accessed on 20 November 2020). 
19. Stremel, T.R.D.O.; Domingues, C.E.; Zittel, R.; Silva, C.P.; Weinert, P.L.; Monteiro, F.C.; Campos, S.X. Development, validation and matrix effect of a QuEChERS method for the analysis of organochlorine pesticides in fish tissue. J. Environ. Sci. Health Part B 2018, 53, 246-254. [CrossRef] [PubMed]

20. Pizzutti, I.R.; de Kok, A.; Hiemstra, M.; Wickert, C.; Prestes, O.D. Method validation and comparison of acetonitrile and acetone extraction for the analysis of 169 pesticides in soya grain by liquid chromatography-tandem mass spectrometry. J. Chromatogr. A 2009, 1216, 4539-4552. [CrossRef] [PubMed]

21. Commission Regulation (EC) No. 835.2011 Amending Regulation (EC) No. 1881/2011 as Regards Maximum Levels for Policy Aromatic Hydrocarbons in Food Stuffs. Off. J. Eur. Union 2011, 215, 4-8. Available online: https: / / eur-lex.europa.eu/LexUriServ / LexUriServ.do?uri=OJ:L:2011:215:0004:0008:EN:PDF (accessed on 20 November 2020).

22. Kumar, B.; Verma, V.K.; Gaur, R.; Kumar, S.; Sharma, C.S.; Akolkar, A.B. Validation of HPLC method for determination of priority polycyclic aromatic hydrocarbons (PAHs) in waste water and sediments. Adv. Appl. Sci. Res. 2014, 5, 201-209.

23. Lee, Y.N.; Shin, H.S. Analytical Method for the Determination of Polycyclic Aromatic Hydrocarbons from Various Ready-to-Eat Food Products in Korea. Polycycl. Aromat. Compd. 2019, 41, 653-662. [CrossRef]

24. Dai, R.; Ren, X.; He, X.; Huo, Y. Convenient analytical method for quantitative determination of 23 pesticide residues in herbs by gas chromatography-mass spectrometry. Bull. Environ. Contam. Toxicol. 2011, 86, 559. [CrossRef]

25. Attallah, E.R.; Barakat, D.A.; Maatook, G.R.; Badawy, H.A. Validation of a quick and easy (QuEChERS) method for the determination of pesticides residue in dried herbs. J. Food Agric. Environ. 2012, 10, 755-762.

26. Chen, L.; Song, F.; Liu, Z.; Zheng, Z.; Xing, J.; Liu, S. Multi-residue method for fast determination of pesticide residues in plants used in traditional Chinese medicine by ultra-high-performance liquid chromatography coupled to tandem mass spectrometry. $J$. Chromatogr. A 2012, 1225, 132-140. [CrossRef]

27. Cui, Z.; Ge, N.; Zhang, A.; Liu, Y.; Zhang, J.; Cao, Y. Comprehensive determination of polycyclic aromatic hydrocarbons in Chinese herbal medicines by solid phase extraction and gas chromatography coupled to tandem mass spectrometry. Anal. Bioanal. Chem. 2015, 407, 1989-1997. [CrossRef]

28. Shi, Z.; Yan, J.; Ma, Y.; Zhang, H. Cloud point extraction-HPLC determination of polycyclic aromatic hydrocarbons residues in traditional Chinese medicinal herbs. Procedia Environ. Sci. 2011, 10, 1216-1221. [CrossRef]

29. Kim, G.Y.; Kim, H.J.; Lee, S.D.; Lee, Y.K.; Yuk, Y.S. Study on the Assessment of PAHs Content and Risk Exposure of Convergence Herbal Pills. J. Korea Converg. Soc. 2018, 9, 75-84.

30. Thi, L.A.P.; Ngoc, N.T.; Quynh, N.T.; Van Thanh, N.; Kim, T.T.; Anh, D.H.; Viet, P.H. Polycyclic aromatic hydrocarbons (PAHs) in dry tea leaves and tea infusions in Vietnam: Contamination levels and dietary risk assessment. Environ. Geochem. Health 2020, 42, 2853-2863. [CrossRef] 